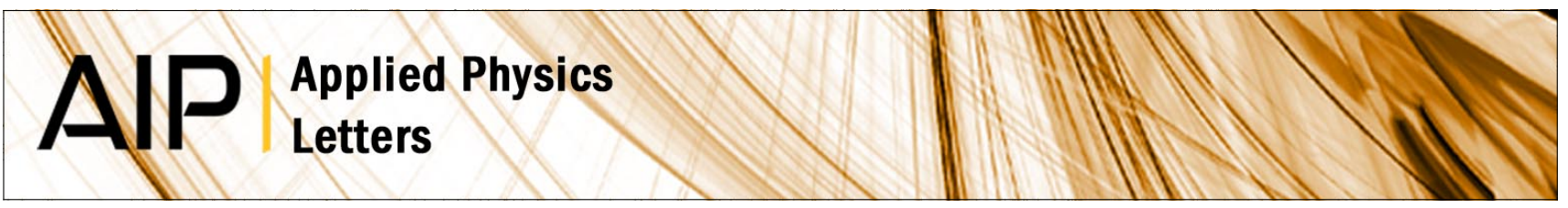

\title{
Plasmon-assisted high reflectivity and strong magneto-optical Kerr effect in permalloy gratings
}

Nikita Kostylev, Ivan S. Maksymov, Adekunle O. Adeyeye, Sergey Samarin, Mikhail Kostylev et al.

Citation: Appl. Phys. Lett. 102, 121907 (2013); doi: 10.1063/1.4798657

View online: http://dx.doi.org/10.1063/1.4798657

View Table of Contents: http://apl.aip.org/resource/1/APPLAB/v102/i12

Published by the AIP Publishing LLC.

\section{Additional information on Appl. Phys. Lett.}

Journal Homepage: http://apl.aip.org/

Journal Information: http://apl.aip.org/about/about_the_journal

Top downloads: http://apl.aip.org/features/most_downloaded

Information for Authors: http://apl.aip.org/authors

\section{ADVERTISEMENT}
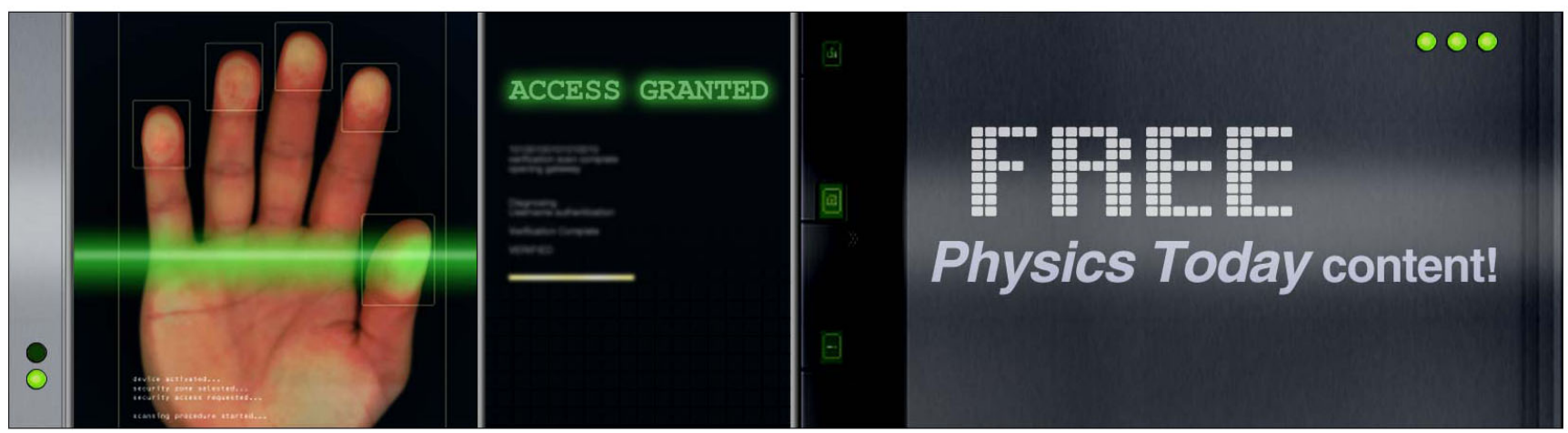


\title{
Plasmon-assisted high reflectivity and strong magneto-optical Kerr effect in permalloy gratings
}

\author{
Nikita Kostylev, ${ }^{1}$ Ivan S. Maksymov, ${ }^{1,2}$ Adekunle O. Adeyeye,${ }^{3}$ Sergey Samarin, ${ }^{1}$ \\ Mikhail Kostylev, ${ }^{1}$ and Jim F. Williams ${ }^{1}$ \\ ${ }^{1}$ School of Physics, University of Western Australia, 35 Stirling Highway, Crawley WA 6009, Australia \\ ${ }^{2}$ Nonlinear Physics Centre, Research School of Physics and Engineering, Australian National University, \\ Canberra ACT 0200, Australia \\ ${ }^{3}$ Information Storage Materials Laboratory, Department of Electrical and Computer Engineering, \\ National University of Singapore, Singapore 117576, Singapore
}

(Received 25 January 2013; accepted 15 March 2013; published online 28 March 2013)

\begin{abstract}
We demonstrate experimentally a strong plasmon-assisted enhancement of the transverse magnetooptical Kerr effect in permalloy gratings. The enhanced transverse magneto-optical Kerr effect is accompanied by an increased grating reflectivity with the maximum of enhancement being correlated with plasmonic Fano resonances. This correlation was confirmed by an intuitive Fano model and also through full-vectorial optical simulations. Simultaneously high reflectivity and transverse magnetooptical Kerr effect as well as narrowest ferromagnetic resonance linewidth and vanishing anisotropy make permalloy nanostructures attractive for applications in spintronics and nano-optics such as, for example, all-optical excitation of propagating spin waves and spectral tuning of optical nanoantennas. ㅇ 2013 American Institute of Physics. [http://dx.doi.org/10.1063/1.4798657]
\end{abstract}

Modern plasmonic devices based on metal nanostructures aim to control the excitation of plasmons, their propagation, dispersion, confinement, and mode structure at a length scale that is much shorter than an optical wavelength. ${ }^{1}$ Since all metals absorb light in the visible and IR spectral ranges, maximum efficiency can be achieved only by using metals with the lowest absorption cross-section. Therefore, most of the plasmonic devices are made of gold or silver because these two metals exhibit the lowest absorption losses at optical frequencies. However, in many practical cases, these metals must be combined with optically active materials in order to provide active control of plasmons. ${ }^{1}$ In particular, plasmons can be controlled by a magnetic field applied to a hybrid device consisting of a plasmonic metal nanostructure combined with a ferromagnetic layer. ${ }^{2-4}$ While the former supports propagating or localized plasmon modes with the lowest possible absorption losses, the latter exhibits a large magneto-optical activity that opens up routes for ultrafast control of light such as, e.g., magneto-plasmonic switching or high-sensitivity biosensing (for a review see, e.g., Ref. 5 and references therein). Similar functionality can be achieved with pure ferromagnetic structures made of, e.g., nickel. ${ }^{6-8}$ Although ferromagnetic metals exhibit a stronger optical damping as compared with gold or silver, ${ }^{7}$ resonance excitation of surface plasmons in ferromagnetic nanostructures makes it possible to enhance the transversal magnetooptical Kerr effect (TMOKE). ${ }^{9}$ The enhancement of TMOKE is important to spintronics and magnetization dynamics. ${ }^{10}$ In particular, the TMOKE can be used as one of the channels of interaction between plasmons and spin waves with the ultimate goal to increase efficiency of optical modulators based on scattering of light on travelling spin waves. ${ }^{11}$ Recently, an all-optical excitation and control of spin waves with a light pulse focused on a magnet have been reported showing that the spin wave wavenumber distribution is diffraction-limited. ${ }^{12}$ This limitation can be circumvented by using magneto-plasmonic nanostructures. Moreover, the TMOKE enables space-resolved observation of fast magnetization dynamics ${ }^{13-15}$ having characteristic frequencies in the gigahertz range. In this case, standing and propagating spin waves ${ }^{16}$ as well as magnetization switching ${ }^{17}$ are typical examples of the magnetic dynamic phenomena that are of paramount importance for microwave signal processing, ${ }^{14}$ magnetic memory, logic, ${ }^{17,18}$ and sensors. ${ }^{19,20}$

Most of these effects have been observed in permalloy $\left(\mathrm{Ni}_{80} \mathrm{Fe}_{20}\right)$ based nanostructures and multilayers. Permalloy is paramount for all these applications because of the optimum combination of magnetic properties: the vanishing magnetic anisotropy and the smallest magnetic (Gilbert) damping among ferromagnetic metals. ${ }^{13-15}$ As a result, magnetization dynamics in plane periodic permalloy nanostructures has attracted a lot of attention and has been largely studied using optical methods in reflection. ${ }^{16}$

Nickel gratings were used in the previous magnetoplasmonics studies. ${ }^{6-8}$ Unfortunately, nickel is characterized by large magnetic losses and is known as a material with very large magnetostriction. Furthermore, its saturation magnetization $(6000 / 4 \pi$ Oe) is small (almost two times smaller than for permalloy and almost four times smaller than for iron). For all these reasons, use of nickel in spintronics and magnetization dynamics is quite limited.

In this letter, we demonstrate experimentally a considerable enhancement of the TMOKE in a permalloy grating in the frequency range of plasmon resonance. This enhancement is accompanied by an increase in grating reflectivity, contrary to the previous experiment (conducted on corrugated nickel gratings) where enhancement of TMOKE was accompanied by a considerable dip in reflectivity. ${ }^{6}$

Permalloy gratings studied in our experiments were fabricated on a silicon substrate by deep UV lithography followed by lift-off. ${ }^{21}$ They represent a periodic array of parallel permalloy nanostripes separated by air gaps [Fig. 1(a)] 

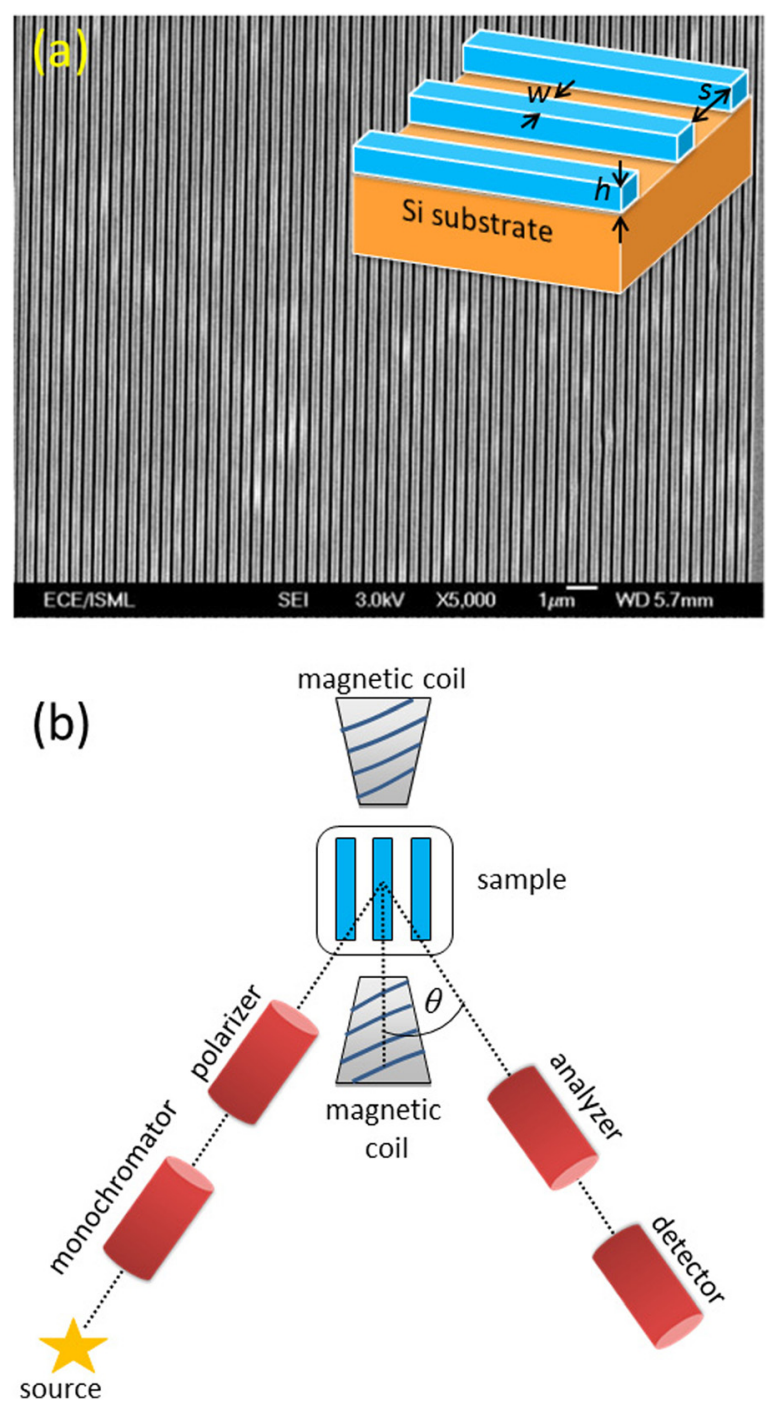

FIG. 1. (a) Scanning electron micrograph and schematic of the investigated permalloy grating. The total area of the grating is $0.5 \times 0.5 \mathrm{~cm}^{2}, h=100 \mathrm{~nm}$, $w=264 \mathrm{~nm}$, and $s=113 \mathrm{~nm}$. The thickness of the Si substrate is $0.8 \mathrm{~mm}$. The gold reference grating has the same dimensions. (b) Schematic of the VASE-based experimental setup with a custom designed electromagnet attachment.

Several samples of this batch were characterized previously with a ferromagnetic resonance method and showed excellent magnetic characteristics. ${ }^{22}$ Reference nonmagnetic gold gratings with the same geometry were also fabricated and optically characterized [Figs. 2(a) and 2(b)]. Reflectance values were measured at three different incident angles over a wide spectral range using a variable angle spectroscopic ellipsometer (VASE), ${ }^{23}$ which gives reflectance as a ratio of the intensities of the incident and reflected beams. Our VASE setup [Fig. 1(b)] uses a standard wide white beam ( $\sim 4 \mathrm{~mm}$ in diameter) generated by a xenon lamp. The beam is passed through a monochromator that adjusts the wavelength to a required value with a bandwidth of $3 \mathrm{~nm}$. The usable spectral range of the device, over which the sampled data have an acceptable error, is approximately $400-800 \mathrm{~nm}$. Data were obtained for both $s$ - and $p$-polarized beams with statistical error of no more than \pm 0.025 .

The reflectivity spectra of the reference gold grating for the $s$-polarization [Fig. 2(b)] are very similar compared to
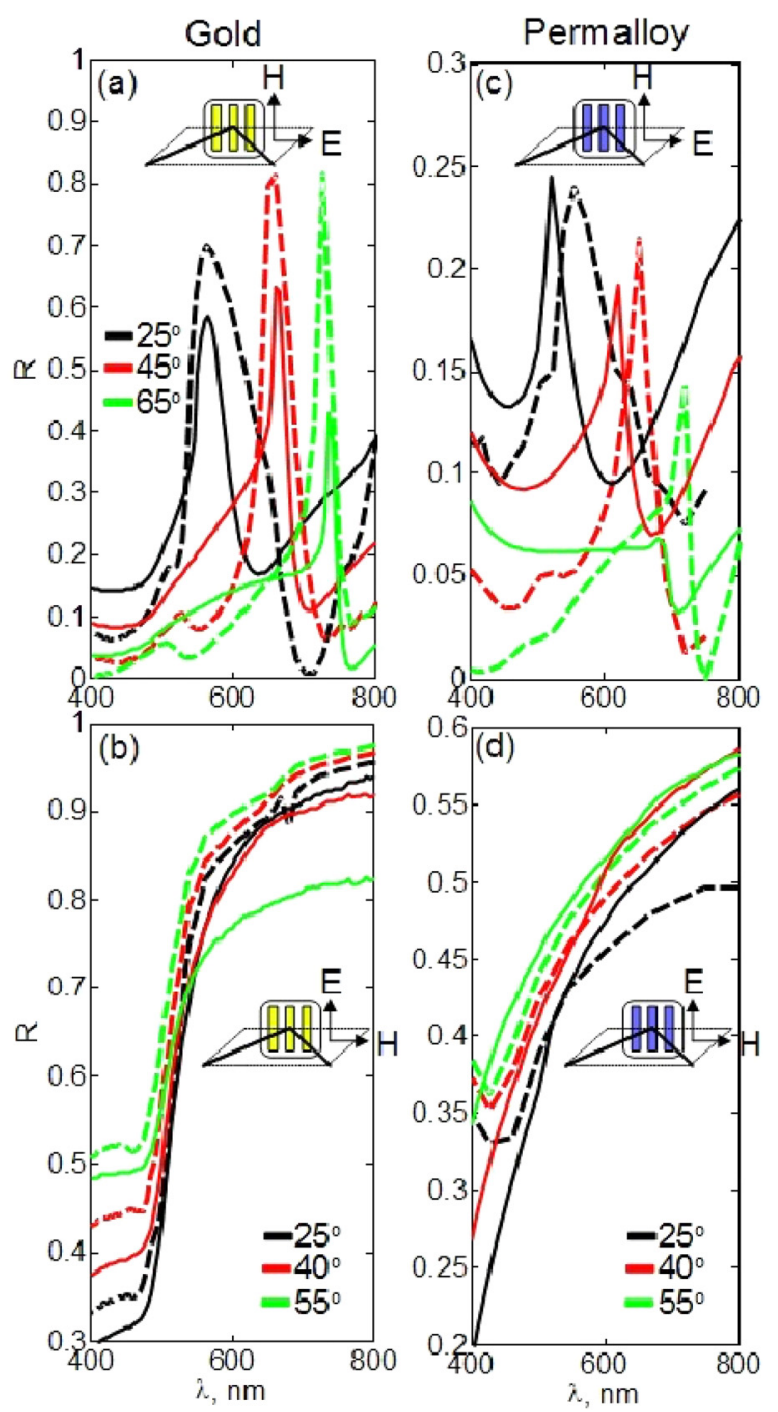

FIG. 2. Measured (solid lines) and simulated (dashed lines) reflection spectra of the $(a, b)$ reference gold grating and $(c, d)$ permalloy grating. The insets show the grating orientation with respect the plane of incidence. Top row-light is $p$-polarized. Bottom row-light is $s$-polarized.

the data obtained for the continuous gold film (not shown) and do not exhibit any special feature. However, the reflection in the $p$-polarization [Fig. 2(a)] is dominated by sharp peaks with asymmetric lineshape typical of Fano resonances. $^{24,25}$ The asymmetric lineshape arises from the constructive and destructive interference of a narrow discrete resonance with a broad spectral line or continuum of modes.

Using MICROWAVE CST STUDIO software implementing a finite-integration technique, we calculated reflectivity spectra of the reference gold grating using refractive index of gold from Ref. 26. The results of our simulations demonstrate consistency of our experimental method and theoretical approach [Figs. 2(a) and 2(b)] in terms of the lineshape of the peaks in the spectra. ${ }^{27}$ We note that the grating also supports guided and evanescent modes that can be seen in the reflectivity spectra as secondary peaks. In our experiment, the contribution of these modes is rather weak because they decay in large area gratings due to fabrication imperfections. However, the secondary peaks can be identified as a peak broadening from the short-wavelength side. Since no imperfections were included in our model, our theoretical spectra 
exhibit stronger secondary peaks at frequencies consistent with the experimentally observed peak broadening.

The reflectivity spectra of ferromagnetic permalloy gratings were measured under the same experimental conditions. CST MICROWAVE STUDIO simulations were conducted using the complex refractive index extracted from measurement data of the continuous permalloy film. Since these data were affected by roughness of the film surface, measures were taken to compensate for artifacts. This ensured a reasonable agreement between the calculations and measurements [Figs. 2(c) and 2(d)]. The spectra of the permalloy grating also exhibit asymmetric Fano resonances [Figs. 2(c) and 2(d)] and are very similar to those of the reference gold grating [Figs. 2(a) and 2(b)]. We note the presence of secondary peaks observed in the spectra of the reference gold grating.

As a next step, we investigate the TMOKE in the permalloy grating. We use $p$-polarized incident light because it remains linearly polarized after reflection if the magnetization is parallel to the surface of the sample and perpendicular to the plane of incidence. The TMOKE is only observable in magnetic structures and it is characterized by the relative change of reflected light intensity $I(M)$ when the magnetization $M$ is reversed $^{9}$

$$
\delta=[I(M)-I(-M)] / I(0) .
$$

The intensity changes due to the magnetic field induced change of the boundary conditions at the surface of a magnetized medium. Magnetized media can be characterized by the permittivity tensor $\varepsilon(M)$ having the following nonzero components: $\varepsilon_{11}=\varepsilon_{22}=\varepsilon_{33}=\varepsilon, \quad \varepsilon_{13}=i g$, and $\varepsilon_{31}=-i g$, where $g$ is the value of the gyration. ${ }^{9}$ If there is absorption, both the $\varepsilon$ and $g$ are complex functions of the frequency. Most significantly, near the surface of a magnetized medium, $g$ is proportional to the cross product between the magnetization vector and the surface normal vector and therefore it changes its sign when the magnetization is reversed. ${ }^{4}$

In order to enable measurements of the TMOKE, the VASE setup was extended by a custom-designed electromagnet attachment with coil windings serving as a source of uniform uniaxial magnetic field [Fig. 1(b)]. The setup was calibrated and the magnetic field strength inside the solenoids was measured with a Gaussmeter to be $250 \mathrm{Oe}$. The TMOKE spectrum of the permalloy grating was recorded in the spectral range of $400-800 \mathrm{~nm}$ at the incidence light angle of $35^{\circ}$ and the magnetic field periodically switched between +250 Oe and -250 Oe. This angle was found to be the optimal setting to minimize diffraction from the edges of the sample as well as to ensure that the light beam is not obscured by the electromagnet.

Figure 3(a) shows the TMOKE response measured on a $100 \mathrm{~nm}$ thick continuous permalloy film (dashed line) and the permalloy grating (solid line). The experiment shows a considerable enhancement of the TMOKE response of the grating at $\lambda \approx 590 \mathrm{~nm}$. We observe simultaneously high reflectivity [see Fig. 3(b)] and TMOKE response. (Recall that in the previous studies, TMOKE enhancement coincided with a dip in reflectivity. ${ }^{6}$ ) Maximization of reflectivity is important for many practical applications such as, for
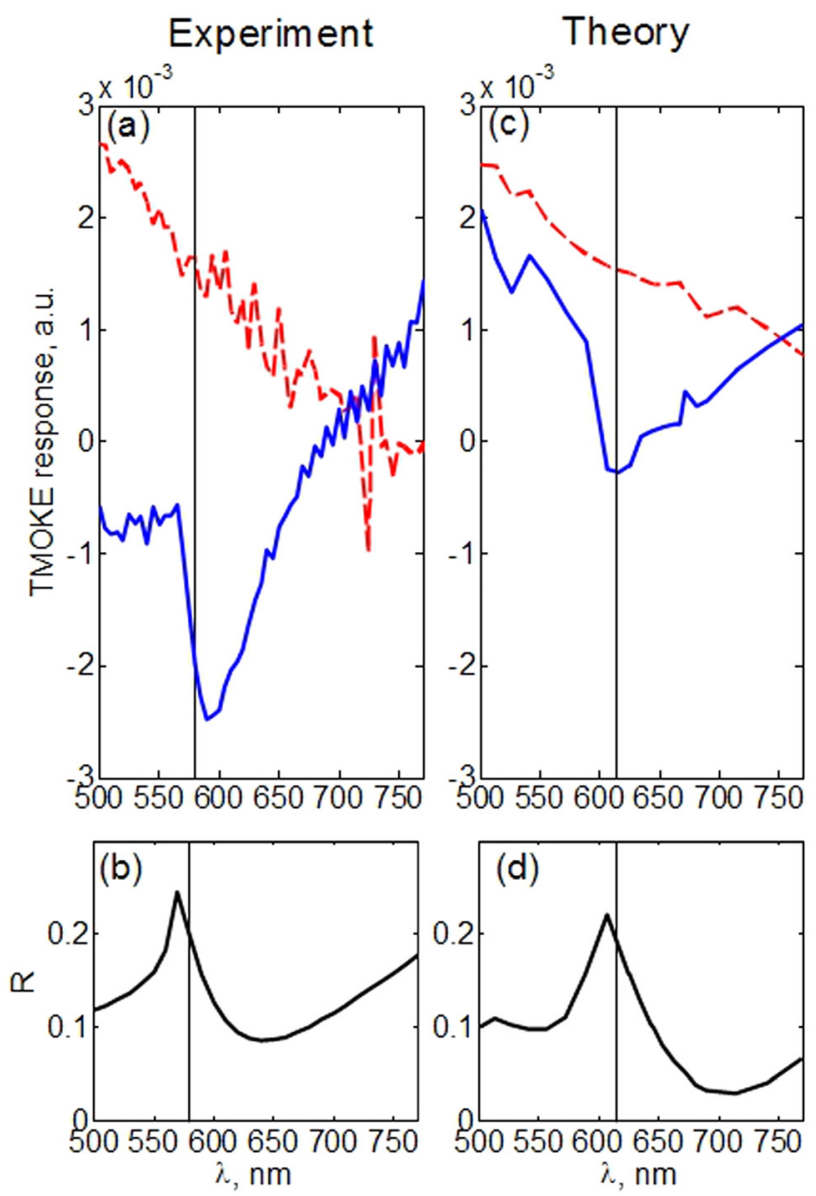

FIG. 3. (a) TMOKE response of the $100 \mathrm{~nm}$ reference permalloy film (dashed line) and permalloy grating (solid line) measured at the incidence angle of $35^{\circ}$ and magnetic field set to +250 Oe and -250 Oe. (b) Measured reflectivity spectrum of the permalloy grating at the incidence angle of $35^{\circ}$. Here and also in the panel (d), the vertical straight solid line denotes the Fano resonance wavelengths. (c) Simulated TMOKE response of the $100 \mathrm{~nm}$ permalloy reference film (dashed line) and permalloy grating (solid line) at the incidence angle of $35^{\circ}$ and at $g= \pm 0.2$. (d) Simulated reflectivity spectrum of the permalloy grating at the incidence angle of $35^{\circ}$. We note that the maximum of the experimentally observed TMOKE enhancement is very close to the maximum slope of the experimental Fano resonance peak where TMOKE is expected to be maximum. This discrepancy is due to inaccuracy of the fitting procedure arising due to noise in experimental data.

example, space-resolved observation of fast magnetization dynamics. ${ }^{13-15}$

We used the Fano resonance profile fitting ${ }^{24}$ in order to demonstrate a correlation between the plasmonic Fano resonance of the permalloy grating and the experimentally observed enhancement of the TMOKE response. A Fano-fit function $F(\omega)=A_{0}+F_{0}\left\{q+2\left(\omega-\omega_{0}\right) / \Gamma\right\}^{2} /\left\{1+\left[2\left(\omega-\omega_{0}\right) / \Gamma\right]^{2}\right\}$ was used to produce best-fit curves that allows the determination of the Fano resonance frequency $\omega_{0}$ and the resonance linewidth $\Gamma$. The parameter $q$ stands for the asymmetry of the Fano lineshape defined as a ratio between the strength of the resonant and nonresonant light reflection mechanisms. ${ }^{24,28}$

An agreement between the resonance frequency found using the Fano-fit function [vertical straight line in Figs. 3(a) and 3(b)] and the maximum of the experimental TMOKE response confirmed that the enhancement in the measured TMOKE response is correlated with the plasmonic resonance of the permalloy grating. This conclusion was further supported by numerical simulations in which the value of the 
gyration $g$ was chosen based on a large number of numerical data and their projection onto experimental results. Even though in practice $g$ is a complex value and a function of the frequency, ${ }^{9}$ our simulations for a fixed value of $g= \pm 0.2$ showed large non-monotonic variation of the simulated TMOKE response of the grating with the wavelength in the vicinity of the Fano resonance. Similar to the experiment, this variation is significantly larger than for the continuous film [Fig. 3(c)]. The simulations also showed a good correlation between the extremum in the simulated TMOKE response (Fig. 3(c)) and the plasmonic resonance of the permalloy grating [straight line in Figs. 3(c) and 3(d)].

The experimental conditions did not allow us measuring the absolute values of reflectivity for the two opposite directions of the magnetization vector with accuracy which is sufficient for reliable extraction of the value of the shift in the Fano resonance frequency upon reversal of magnetization. Using the simulation data, we estimated this shift to be of at least $1.5 \mathrm{~nm}$. Graphical analysis of changes in the grating reflectivity caused by this shift and the interpretation of the origin of the TMOKE enhancement are difficult because the corresponding curves coincide to the graphical accuracy. Therefore, for the sake of clarity, in Fig. 4, we analyze a
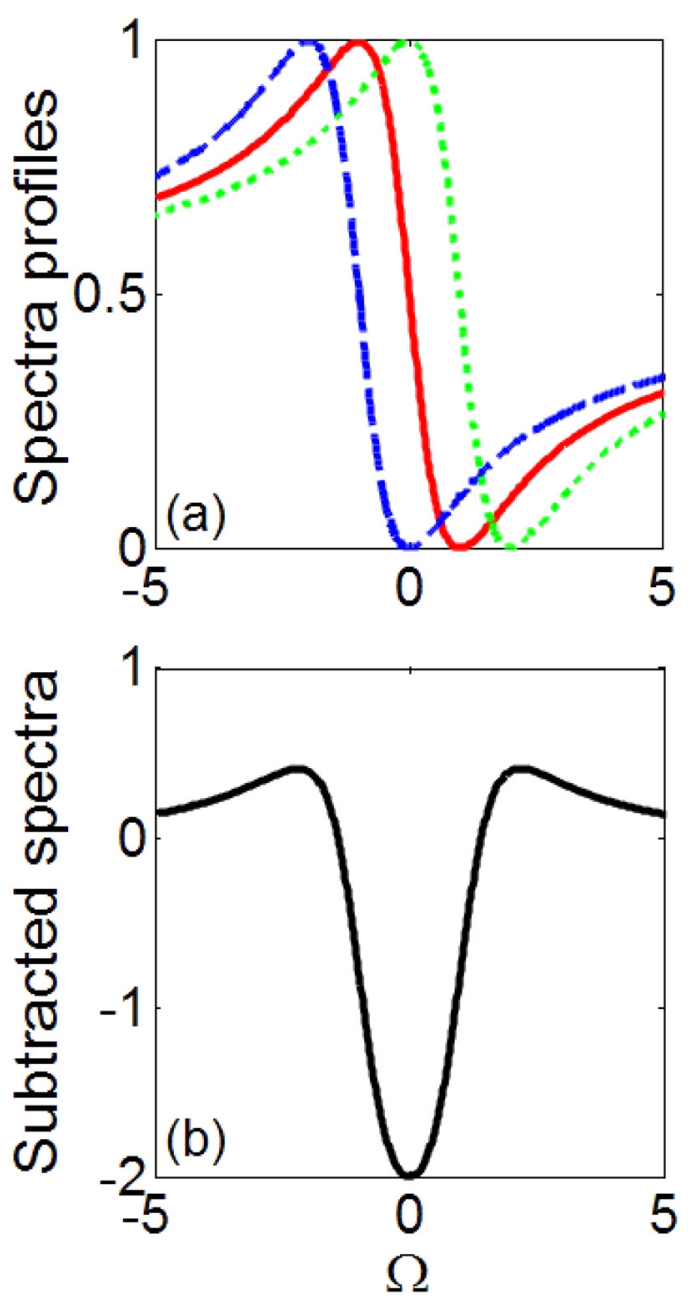

FIG. 4. Schematic demonstration of the effect of plasmon resonance shift on the TMOKE. (a) Solid line-normalized Fano spectra profile with the prefactor $1 /\left(1+q^{2}\right)$ for the asymmetry parameter $q=1$. Dashed and dotted lines-shifted Fano profiles illustrating the effect of the magnetization direction. (b) Subtracted spectra mimicking the TMOKE response. model of general asymmetric Fano resonance profile with magnified characteristic features described by the formula $\sigma=(\Omega+q)^{2} /\left(\Omega^{2}+1\right)$ with $\Omega$ being the reduced frequency defined as $2\left(\omega-\omega_{0}\right) / \Gamma{ }^{24}$ We deliberately choose $q=1$ because in this case, the Fano resonance frequency is located exactly at half the distance between the minimum and the maximum of the profile ${ }^{24}[\Omega=0$ in Fig. 4(a)]. The resonance frequency of the plasmon resonance changes as the direction of the magnetization is flipped. This change shows up as the maximum of the TMOKE when the reflectivity curves for the opposite directions of magnetization vector are subtracted [Fig. 4(b)]. For the chosen Fano parameter $(q=1)$, this occurs at $\Omega=0$, where the slope of the Fano resonance peak is maximum.

The theory of Belotelov et al. ${ }^{4}$ explains the TMOKE enhancement as a modification of the light-plasmon coupling in the presence of the external magnetic field. The result we obtain with the simple Fano resonance model is consistent with this model. Furthermore, in numerical simulations, we observed that the relative gain in the reflectivity for the negative values of $g$ is smaller than the relative loss observed for the positive values. This also agrees with the theory predicting an optical nonreciprocity due to a symmetry breaking at the interface of a transversely magnetized medium. ${ }^{4,29}$

In summary, we demonstrated convincingly that a permalloy magneto-plasmonic grating can efficiently support plasmonic resonances leading to a considerable enhancement of the TMOKE response. This enhancement is also accompanied by an increase in grating reflectivity. Despite a larger resonance linewidth as compared with that of the reference gold grating of the same geometry, the magnetically controlled plasmonic effects in permalloy may be a viable way to enhance the efficiency of light interaction with microwave spin waves. The observed enhancement of TMOKE also opens up other exciting opportunities in increasing efficiency of those magneto-electronic devices whose functionality requires optical reading of the magnetic state of permalloy nano-objects. We also anticipate the application of permalloy in subwavelength plasmonic devices such as tunable optical nanoantennas ${ }^{30}$ controlled by external magnetic fields.

This work was supported by the Australian Research Council and the Ministry of Education, Singapore. I.S.M. gratefully acknowledges a postdoctoral research fellowship from the University of Western Australia. The authors thank Yuri Kivshar from the Australian National University for valuable comments.

${ }^{1}$ M. I. Stockman, Opt. Express 19, 22029 (2011).

${ }^{2}$ V. V. Temnov, G. Armelles, U. Woggon, D. Guzatov, A. Cebollada, A. Garcia-Martin, J.-M. Garcia-Martin, T. Thomay, A. Leitenstorfer, and R. Bratschitsch, Nat. Photonics 4, 107 (2010).

${ }^{3}$ V. I. Belotelov, I. A. Akimov, M. Pohl, V. A. Kotov, S. Kasture, A. S. Vengurlekar, A. V. Gopal, D. R. Yakovlev, A. K. Zvezdin, and M. Bayer, Nat. Nanotechnol. 6, 370 (2011).

${ }^{4}$ V. I. Belotelov, I. A. Akimov, M. Pohl, A. N. Kalish, S. Kasture, A. S. Vengurlekar, A. V. Gopal, V. A. Kotov, D. Yakovlev, A. K. Zvezdin, and M. Bayer, J. Phys.: Conf. Ser. 303, 012038 (2011).

${ }^{5}$ V. V. Temnov, Nat. Photonics 6, 728 (2012).

${ }^{6}$ A. A. Grunin, A. G. Zhdanov, A. A. Ezhov, E. A. Ganshina, and A. A. Fedyanin, Appl. Phys. Lett. 97, 261908 (2010). 
${ }^{7}$ J. Chen, P. Albella, Z. Pirzadeh, P. Alonso-González, F. Huth, S. Bonetti, V. Bonanni, J. Åkerman, J. Nogués, P. Vavassori, A. Dmitriev, J. Aizpurua, and R. Hillenbrand, Small 7, 2341 (2011).

${ }^{8}$ V. Bonanni, S. Bonetti, T. Pakizeh, Z. Pirzadeh, J. Chen, J. Nogués, P. Vavassori, R. Hillenbrand, J. Åkerman, and A. Dmitriev, Nano Lett. 11, 5333 (2011).

${ }^{9}$ A. K. Zvezdin and V. A. Kotov, Modern Magneto-Optics and MagnetoOptical Materials (Taylor \& Francis Group, NY, 1997).

${ }^{10}$ A. Kirilyuk, A. V. Kimel, and Th. Rasing, Rev. Mod. Phys. 82, 2731 (2010).

${ }^{11}$ V. V. Matiushev, M. P. Kostylev, A. A. Stashkevich, and J. M. Desvigne, J. Appl. Phys. 77, 2087, (1995).

${ }^{12}$ T. Satoh, Y. Terui, R. Moriya, B. A. Ivanov, K. Ando, E. Saitoh, T. Shimura, and K. Kuroda, Nat. Photonics 6, 662 (2012).

${ }^{13}$ J. P. Park, P. Eames, D. M. Engebretson, J. Berezovsky, and P. A. Crowell, Phys. Rev. Lett. 89, 277201 (2002).

${ }^{14}$ V. V. Kruglyak, S. O. Demokritov, and D. Grundler, J. Phys. D.: Appl. Phys. 43, 264001 (2010).

${ }^{15}$ T. Verduci, C. Rufo, A. Berger, V. Metlushko, B. Ilic, and P. Vavassori, Appl. Phys. Lett. 99, 092501 (2011).

${ }^{16}$ G. Gubbiotti, S. Tacchi, M. Madami, G. Carlotti, A. O. Adeyeye, and M. Kostylev, J. Phys. D: Appl. Phys. 43, 264003 (2010).

${ }^{17}$ D. A. Allwood, G. Xiong, C. C. Faulkner, D. Atkinson, D. Petit, and R. P. Cowburn, Science 309, 1688 (2005).
${ }^{18}$ J. Åkerman, Science 308, 508 (2005).

${ }^{19}$ M. Inoue, A. Baryshev, H. Takagi, P. B. Lim, K. Hatafuku, J. Noda, and K. Togo, Appl. Phys. Lett. 98, 132511 (2011).

${ }^{20}$ C. S. Chang, M. Kostylev, and E. Ivanov, "Metallic spintronic nanofilm as a hydrogen sensor," Appl. Phys. Lett. (to be published); e-print arXiv: 1301.1106.

${ }^{21}$ A. O. Adeyeye and N. Singh, J. Phys. D: Appl. Phys. 41, 153001 (2008).

${ }^{22}$ C. S. Chang, M. Kostylev, A. O. Adeyeye, M. Bailleul, and S. Samarin, Europhys. Lett. 96, 57007 (2011).

${ }^{23}$ VASE Hardware Manual (J.A. Woolam Co., Inc., Lincoln, USA, 1996).

${ }^{24}$ A. E. Miroshnichenko, S. Flach, and Yu. S. Kivshar, Rev. Mod. Phys. 82, 2257 (2010).

${ }^{25}$ A. Hessel and A. A. Oliner, Appl. Opt. 4, 1275 (1965).

${ }^{26}$ E. D. Palik, Handbook of Optical Constants of Solids (Academic Press, New York, 1985).

${ }^{27}$ W. L. Barnes, J. Opt. A, Pure Appl. Opt. 11, 114002 (2009).

${ }^{28}$ I. S. Maksymov and A. E. Miroshnichenko, Opt. Express 19, 5888 (2011).

${ }^{29}$ G. Armelles, A. Cebollada, A. García-Martín, and M. Ujué González, Adv. Opt. Mater. 1, 10 (2013).

${ }^{30}$ I. S. Maksymov, A. E. Miroshnichenko, and Yu. S. Kivshar, Opt. Express 20, 8929 (2012). 